\title{
SPECIAL COMMUNICATION
}

\section{Upgrade on the orthopedic approach in Charcot-Marie-Tooth Diseases}

\section{Actualización en el abordaje ortoprotésico en las Enfermedades de Charcot-Marie- Tooth}

\author{
Thais Pousada García ${ }^{1}$, Jessica Garabal-Barbeira ${ }^{2}$, Betania Groba ${ }^{1}$, Laura Nieto Riveiro ${ }^{1}$, Javier Pereira Loureiro ${ }^{1}$, Alejandro \\ Pazos Sierra ${ }^{1}$ \\ ${ }^{1}$ Departamento Ciencias da Saúde. Facultade Ciencias da Saúde Universidade da Coruña, A Coruña, España \\ ${ }^{2}$ Departamento de Psicología de la Salud, Universidade da Coruña, A Coruña, España \\ * Correspondence: Thais Pousada García. Departamento Ciencias da Saúde. Facultade Ciencias da Saúde Universidade da Coruña. tpousada @udc.es
}

\begin{abstract}
Objectives: This paper aims to provide to offer a synthesis about the specifications of the intervention and approach to treat the complications of the foot in people affected by Charcot-Marie-Tooth disease.

Methods: A bibliographic search was performed in PubMed and Scopus databases, while relevant information about state of the art from guidelines for intervention in neuromuscular diseases was selected.

Results: The main characteristics and consequences of the disease, the associated complications and the intervention's techniques have been exposed. Splints, orthoses and footwear - orthopedic, are essential complements to offer a comprehensive treatment to the user, in order to achieve a greater degree of personal autonomy.

Conclusions: All the professionals of the interdisciplinary team must to know the characteristics of the disease and the limitations that these cause in the activities of daily living, in order to offer an attention that has a favorable impact on the quality of life.
\end{abstract}

Key Words: Charcot-Marie-Tooth disease, foot orthoses, splints, orthotic devices.

\section{Resumen}

Objetivos Este artículo pretende ofrecer una síntesis acerca de las principales características de la intervención y abordaje de las complicaciones del pie en personas afectadas por las enfermedades de Charcot-Marie-Tooth.

Material y Métodos: Se realizó una búsqueda bibliográfica en las bases Pubmed y Scopus, seleccionando, a la vez, información relevante sobre el estado del arte en guías de intervención en enfermedades neuromusculares.

Resultados: Se exponen las principales características y repercusiones de la enfermedad, complicaciones asociadas y las técnicas de intervención. Los productos de apoyo, ortesis y calzado ortopédico son complementos indispensables para ofrecer un tratamiento integral al usuario, con el fin de alcanzar un mayor grado de autonomía personal.

Conclusiones: Los profesionales del equipo interdisciplinar han de conocer las características de estas enfermedades y las limitaciones que éstas provocan en las actividades de la vida diaria para poder ofrecer una atención que repercuta favorablemente en la calidad de vida.

Palabras Clave: Enfermedades de Charcot-Marie-Tooth, ortesis de pie, férulas, aparatos ortopédicos.

Received: 7 Jun 2017; Acept: 7 Nov 2017.

Conflictos de Interés

Ninguno Declarado.

Fuentes de Financiación

Ninguno Declarado. 


\section{Introducción}

El término "Enfermedad de Charcot-MarieTooth" (CMT) denomina a un grupo de más de 15 enfermedades crónicas del nervio periférico (neuropatías) (1), con una prevalencia que afecta a 1 de cada 2.500 personas (2).

Este conjunto de patologías también se conoce comúnmente como "neuropatía motora y sensorial hereditaria" (HSMN), haciendo referencia a sus dos características principales: presenta un componente genético y hereditario, y afecta a los nervios periféricos y sensoriales. La enfermedad de CMT no describe a un único trastorno, sino un grupo de condiciones que comparten características similares. Por ello, en el momento de proponer una estrategia terapéutica, es importante determinar qué tipo de CMT padece la persona, mediante la elaboración de un completo examen de la historia familiar, y la realización de estudios de conducción nerviosa y genéticos (3).

Entre las principales características clínicas de CMT destacan la debilidad y el desgaste de músculos de miembros inferiores (MMII), especialmente por debajo de las rodillas y, a menudo de las manos. Como componente sensorial, muchas personas también pueden tener pérdida de sensibilidad en las manos y pies (3). Su debut suele manifestarse antes de los 20 años $(3,4)$.

\section{Etiología y Diagnóstico}

El componente neuropático de CMT produce una afectación a nivel de fibras nerviosas, bien en la vaina de mielina que recubre el nervio, o en el propio axón (5). Este daño dificulta la conducción de la señal eléctrica, desde el cerebro al músculo, dando por resultado debilidad muscular y disminución de sensibilidad $(3,4)$. Se han establecido dos tipos principales de CMT (tipos 1 y 2) según qué parte del nervio está afectada. En CMT tipo 1, la alteración se produce a nivel de la vaina de mielina, y en el tipo 2 , del axón.
Estos dos grupos principales se dividen en subtipos diferentes, dependiendo del patrón de herencia y gen involucrado. Se han identificado más de 45 genes cuyas mutaciones causan CMT (3). El tipo de herencia puede ser autosómica dominante o autosómica recesiva (se necesita tener dos copias del gen mutado para mostrar síntomas) (3).

El diagnóstico se establece mediante el examen físico (sintomatología clínica), complementado con estudios electromiográficos (EMG), el análisis genético de una muestra de sangre $\mathrm{y}$, en ocasiones, para confirmación, la biopsia del nervio (6).

\section{Manifestaciones y Evolución}

Como se ha comentado, las principales estructuras corporales afectadas, tanto en movilidad como en sensibilidad, son los MMII y principalmente, los pies. Debido a la amiotrofia y debilidad muscular distal, estos adquieren una actitud en cavo, los dedos se contraen en forma de "garra" y se hace imposible el apoyo sobre la parte delantera del pie para caminar. Los músculos de flexión dorsal son débiles, lo que, unido a las retracciones tendinosas, obliga a elevarlo a una altura mayor para que la punta no tropiece con el suelo (1). Estos primeros signos son los principales responsables de las deformaciones ortopédicas. Sin embargo, la capacidad de marcha no suele perderse, aunque algunas personas (especialmente en edades avanzadas) necesitan productos de apoyo (PA) para caminar (3).

Otros síntomas frecuentes son la fatigabilidad, inestabilidad del equilibrio, entumecimiento de extremidades (aunque raramente es severo) y dificultad para mantener la posición erguida o subir escaleras. La afectación de las manos es generalmente tardía, y aparece sólo tras años de evolución de la enfermedad. Las funciones vitales e intelectuales no se ven alteradas (1). 
Su evolución suele ser muy variable, según el tipo, subtipo y factores individuales. La esperanza de vida no se modifica y es posible mantener una actividad sociolaboral adaptada, en la mayor parte de los casos (1).

\section{Tratamiento y propuestas de intervención en CMT}

Actualmente, no existe tratamiento curativo específico para la CMT, pero ello no significa que las personas afectadas no requieran una intervención rehabilitadora. Por ello, se considera fundamental un enfoque multidisciplinar que combine fisioterapia, terapia ocupacional, la actividad física y el abordaje ortopédico para mantener la capacidad funcional de las personas afectadas durante el mayor tiempo posible.

El tratamiento ortopédico incluye la valoración de los componentes estructurales y fisiológicos alterados, así como la elaboración y prescripción de ortesis específicas, recomendación de calzado ortopédico y acciones preventivas, en combinación con un abordaje fisioterapéutico, que ha de ser precoz, regular e individualizado. Esta intervención facilitará que la progresión sea más lenta, ya que permite mantener la flexibilidad de las articulaciones $(1,7)$.

En algunos casos, cuando la deformidad es intensa y no se puede controlar con las férulas, se recurre al tratamiento quirúrgico. Se considera una opción útil, ya sea para reducir el arco y retracción los dedos de los pies, o para fusionar algunos de huesos del tarso y metatarso (3). La cirugía ortopédica se propone a fin de mantener, o incluso restaurar, la función de caminar $(4,6)$. Las intervenciones más frecuentes se realizan a nivel de tobillos, pero también pueden abarcar otras articulaciones, como cadera y rodillas.
El tratamiento quirúrgico permite reducir los dolores derivados del posicionamiento, prolongar la marcha (con o sin la ayuda de aparatos ortopédicos), facilitar la bipedestación y la sedestación (6), contribuyendo a mejorar la calidad de vida de la persona. No obstante, es necesario que, tras la cirugía, se proporcione una intensa intervención rehabilitadora.

De manera paralela a estas intervenciones, se propone el uso de PA. Estos permiten compensar las dificultades en algunas funciones, aumentando la independencia funcional, tanto en actividades de la vida cotidiana como en los desplazamientos y participación social. Entre los PA más empleados, destacan las ayudas para la marcha y movilidad, como bastón, andador, scooter, silla de ruedas eléctrica, o productos para transferencias, y otros más específicos como cubiertos adaptados, dispositivos de acceso al ordenador, o soportes para brazos (8). Cada persona utiliza estas ayudas según sus necesidades individuales en cada momento, siendo asesorada por un terapeuta ocupacional para la selección de los dispositivos más adecuados a su situación (9).

Características del pie en las Enfermedades de CMT y evolución de sus alteraciones

La falta y/o el desajuste de fuerza entre los músculos en la CMT, así como su tendencia, y la de los tendones, hacia el acortamiento, favorece la aparición de deformidades articulares.(8)

A continuación, se exponen las alteraciones estructurales del pie y tobillo más comunes en CMT:

- Retracciones de músculos y tendones: La atrofia del músculo lleva a que éste pierda fuerza y elasticidad. No todos los músculos se debilitan al mismo nivel, y ello provoca un desequilibrio de la fuerza ejercida entre los agonistas y antagonistas que movilizan una misma articulación. 
Las tensiones contrapuestas favorecen el acortamiento de uno de los músculos y el debilitamiento del otro, de forma progresiva. Esta evolución da lugar a lo que se denominan retracciones musculotendinosas, siendo uno de los objetivos de la prescripción ortopédica precoz, la limitación en la evolución de las mismas (8).

- Deformidades articulares: La disminución del movimiento, producida por dicha debilidad muscular, lleva a que las propias articulaciones también sufran sus consecuencias y tiendan a anquilosarse. La prevalencia de las retracciones, así como los cambios posturales poco frecuentes, fijan las articulaciones en una mala posición, provocando su deformidad (8).

La evolución típica de la pérdida de fuerza muscular lleva a lo que se conoce como "efecto trípode": en la posición estática de soporte del peso corporal, la rigidez del primer vientre del flexor plantar produce una torsión en inversión del pie, provocando una actitud en varo del calcáneo (10).

Como consecuencia de estas alteraciones estructurales, se han identificado varias tipologías, según las características de los pies en personas con CMT (11):

- Pie cavo: Es la postura más común, siendo característico el pie con un arco elevado, y una curvatura rígida bajo el empeine. El resultado de esta actitud postural da lugar a la generación de callosidades, aumento de la incidencia de esguinces de tobillo, y patrón del pie en equino. Los músculos intrínsecos del pie se atrofian, dando lugar a un retraimiento de los dedos. Entre las opciones terapéuticas se incluyen ortesis y cirugía. Las figuras 1 y 2 ejemplifican la estructura y retracciones características del pie cavo en una persona con CMT.

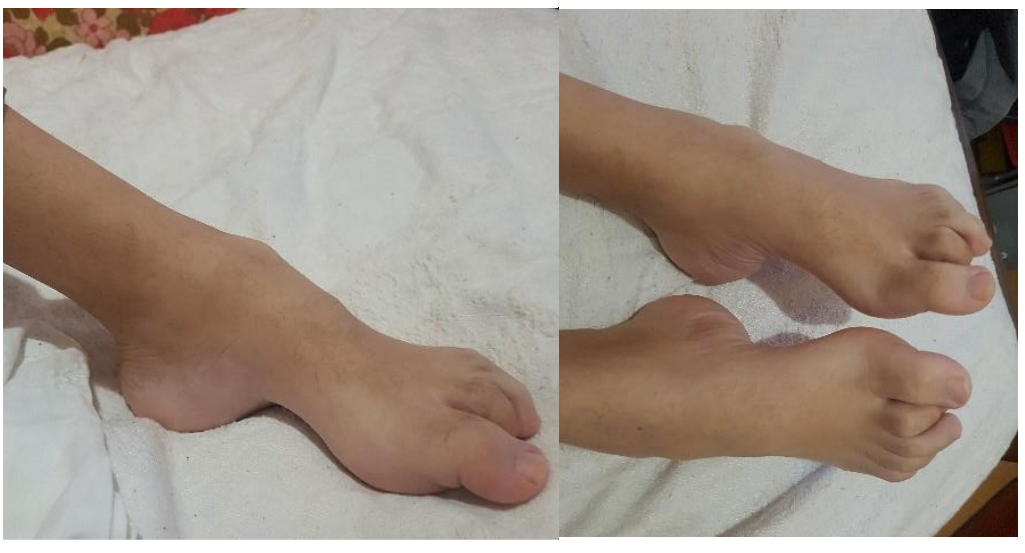

Figuras 1 y 2. Vista Lateral y Dorsal de Pie Cavo.

- Pie plano paralítico: Es menos común y normalmente requiere una fusión quirúrgica en el arco para frenar la progresión del deterioro.

- Caída del pie: Provocada por la debilidad de los músculos tibial anterior, extensor largo de los dedos y extensor del pulgar. La intervención en estos casos contempla la potenciación muscular a través de ejercicios activo/pasivos, el uso de zapatos de base redondeada y cirugía.

Teniendo presente lo anterior, se evidencia que la prescripción ortoprotésica permite limitar o compensar las consecuencias del déficit muscular por medio de diferentes métodos. Una atención precoz, personalizada y continuada contribuye a mantener la autonomía en diferentes áreas y entornos del individuo $\mathrm{y}$, por tanto, a mejorar significativamente la calidad de vida (8).

\section{La importancia de la prevención}

Uno de los puntos clave en el abordaje de las complicaciones de CMT es la anticipación y detección de éstas, y la aplicación de las medidas pertinentes para prevenirlas. 
Además de las consecuencias motoras y anatómicas, se recuerda que la enfermedad de CMT puede provocar entumecimiento y una importante alteración en la sensibilidad. Por ello, pueden surgir serios problemas cutáneos como úlceras, gangrena o incluso infecciones (por ejemplo, pie de atleta). Por ello, las recomendaciones al paciente están orientadas a la observación diaria de la piel, vigilancia y detección de signos de alerta, así como la realización de una buena higiene e hidratación de los pies $(2,11)$.

Teniendo en cuenta esta característica, y ante el riesgo de roces, cortes o heridas punzantes, no se recomienda a las personas afectadas el ir descalzos, por lo que deben decantarse por un zapato cerrado, firme y que permita la transpiración adecuadamente.

La prevención está enfocada también a la conservación de la movilidad, flexibilidad y fuerza. Así, los usuarios han de conocer y aplicar pautas básicas de ejercicios activos y pasivos. La hidroterapia puede ser una estrategia de elección, pero su evidencia no está claramente demostrada, o ésta deriva de estudios observacionales (12).

Previo a la propuesta de cualquier intervención, es preciso realizar una buena evaluación de la situación funcional de la persona. Dicha valoración ha de contemplar (10):

- Historia clínica: Ocupación, actividades actuales, actividades pasadas. Necesidades (qué es lo que más cuesta hacer, cuáles son sus límites) y principales afectaciones: debilidad, inestabilidad, balance muscular y disminución de la capacidad funcional.

- Valoración de la afectación anatómica y cinésica: Análisis de la sensibilidad, rango de movimiento, fuerza muscular, balance corporal, desviaciones y cadencia de la marcha.
- Concreción con el usuario: Posibilidades y estrategias de intervención, objetivos del tratamiento, opciones de uso de ortesis y/o calzado ortopédico y abordaje quirúrgico, en último caso.

\section{Uso de productos ortésicos como recurso clave en la intervención}

Las ortesis o férulas son esenciales para prevenir y corregir las deformidades, así como para conservar la flexibilidad y el equilibrio articular. $\mathrm{Su}$ prescripción y uso ha de ser precoz (ya que es más doloroso cuando las alteraciones están establecidas) y continuo, limitando la aparición de acortamientos y ralentizando la evolución de las alteraciones articulares $(8,12)$. Otras ortesis facilitan los movimientos al mantener las articulaciones en una posición funcional (8). La experiencia en la atención a estos pacientes evidencia un beneficio real para el aparato osteoarticular y muscular. Más allá del aspecto preventivo, se experimenta un mayor bienestar en estas estructuras (8).

La aplicación de productos ortésicos mejora, por tanto, las capacidades funcionales, especialmente en movilidad y desplazamiento, de la persona. No obstante, los profesionales deben tener en cuenta que estos dispositivos no van a corregir totalmente la debilidad y/o desequilibrio de fuerza muscular, y a veces, puede ser complicado establecer el momento concreto y la cantidad de corrección que se ha de aplicar (13). Por otra parte, los estudios evidencian que las personas con CMT tienden a retrasar el uso de las ortesis, especialmente las de tobillo (AFOs) hasta que su capacidad para caminar está tan deteriorada que la elección comienza a parecer inevitable. Esta transición impone cargas propias, por supuesto, incluyendo una evidencia de responsabilidad sobre el profesional (14). 
Durante la infancia y la adolescencia, el objetivo del uso de ortesis es preservar los logros y evitar las deformidades, considerando el crecimiento del infante, y manteniendo la flexibilidad y elasticidad muscular. En la edad adulta, se pretende mantener el cuidado articular, muscular y funcional, así como conseguir el máximo bienestar posible (8).

La temporalidad en su uso (de forma nocturna, diurna o ambos) va a depender de la edad de la persona y del grado de deformidad. Durante la noche, al estar confeccionadas como férulas de reposo, se aprovecha el sueño y el estado de relajación para mantener la articulación afectada en la situación postural óptima. Si se emplean durante el día (ortesis funcional), asiste al movimiento y complementa la acción de las férulas nocturnas y del patrón de ejercicios. Las actuales ortesis para la marcha pueden usarse con unos zapatos clásicos (8).

El médico rehabilitador, quién coordina el equipo multidisciplinar, es el que prescribe las ortesis, con sus características definidas según la evaluación previa y realiza el seguimiento. Posteriormente, el técnico ortoprotésico fabrica las ortesis a medida, a partir de un molde de la zona afectada, verificando la adaptación del dispositivo con varias pruebas.

Las ortesis deben modificarse y reemplazarse para adaptarlas a los cambios anatómicos del usuario. Cualquier producto ortopédico requiere una atenta vigilancia para detectar la aparición de rojeces o de dolores derivados de la presión (8).

\section{Ortesis más frecuentes utilizadas en CMT}

A continuación, se muestran los principales productos ortopédicos empleados por personas con CMT (12):
- Tobilleras: Son muy flexibles y poco restrictivas en el movimiento, pero pueden ofrecer un soporte para evitar la inestabilidad, en caso de que existiera, a nivel de tobillos (15). La figura 3 muestra una tobillera empleada por una persona con CMT, para la que se ha realizado una adaptación con cordón elástico, a fín de evitar la caída del pie durante la fase de balanceo de la marcha.

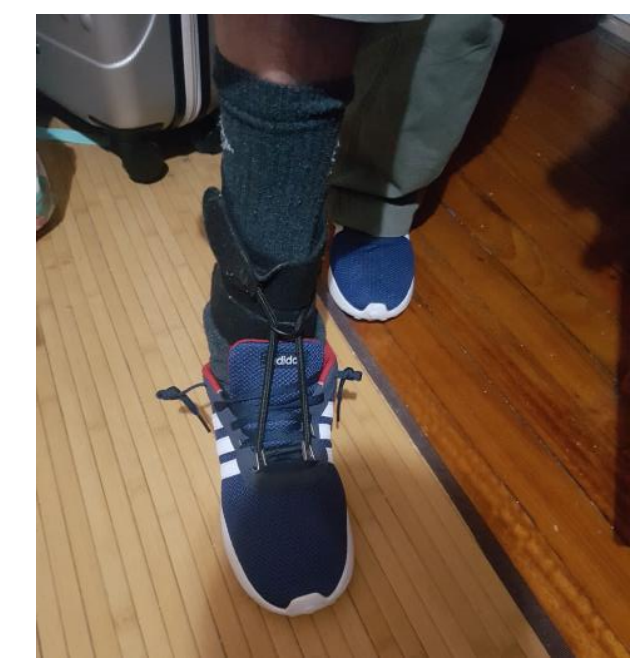

Figura 3. Vista Dorsal de Pie Cavo con tobillera.

- Ortesis de pie y tobillo (AFO): Son las llamadas férulas antiequino, que compensan la caída del pie durante la marcha, ofreciendo un apoyo adecuado a la articulación, y mejoran la estabilidad y posición del pie. Las AFO se pueden emplear durante el sueño, como férulas de reposo, para mantener la longitud del tendón de Aquiles, si existe pérdida de dorsiflexión, en combinación con los estiramientos diarios. En caso de programar su uso diurno, la experiencia clínica sugiere que deben ser prescritas una vez que la deambulación está muy deteriorada, para prevenir las contracturas dolorosas y la deformidad del pie (12). 
Como desventaja, cabe destacar que no tiene sistema de sujeción en la parte inferior, por encima del tobillo, lo que puede crear desajuste ortesis-pie, a la vez que puede provocar rozamiento y calentamiento ante la falta de transpiración (16).

- Ortesis de rodilla, tobillo y pie (KAFO): Aunque su uso no está tan extendido en CMT, suelen recomendarse para prolongar la deambulación y también pueden ayudar a retrasar el inicio de las contracturas de las extremidades inferiores (12), usualmente utilizada para ayudar a caminar. Uno de sus inconvenientes es su gran superficie de contacto, que genera calor y sudoración constante (16).

\section{Evidencia sobre el uso de ortesis en CMT}

En un estudio reciente de Wegener et al (17) en pacientes con CMT se ha demostrado que, en comparación con el uso único de zapato ortopédico, las ortesis aumentan la eversión y la flexión plantar, aumentan la eversión del tobillo $\mathrm{y}$ producen pequeños, pero significativos cambios en rodilla y cadera, indicando un aumento de la rotación interna. Las ortesis fueron calificadas por los participantes como más cómodas, con mayor amortiguación y estabilidad.

En el estudio de Guillebastre et al (18), se determina que varios modelos de AFOs contribuyen a mejorar, en diferentes proporciones, la postura y el control de la marcha, en personas con CMT. En dicho trabajo de casos y controles, valoraron las posibles diferencias entre los zapatos cotidianos, las AFO elásticas y las AFO rígidas. Estas últimas fueron las que demostraron mejores resultados en el incremento del control postural y de la marcha, mientras que las elásticas tuvieron mayor efecto sobre el control dinámico de la deambulación (19).
Por su parte, Rose et al (20), trataron de verificar si la aplicación de un soporte ortésico nocturno (durante 4 semanas), en comparación con estiramientos de los músculos sóleo y gemelo, tenían un efecto positivo sobre el rango de dorsiflexión del tobillo. Sus resultados manifiestan que las ortesis nocturnas aumentaban $4^{\circ}$ este movimiento, y los estiramientos mejoraron hasta $3^{\circ}$ dicho rango.

El efecto de las férulas nocturnas para la extensión de los flexores plantares en el rango de la articulación del tobillo, en personas con CMT, fue analizada por el estudio de Refshauge (21). Sus resultados mostraron que el uso de esta ortesis nocturna no producía diferencias significativas en el rango o fuerza de los movimientos de inversión y eversión.

Otro trabajo trató de determinar las consecuencias del uso de 3 tipos de AFO (tobillera, PushBrace y Multifit Achilles) para el control distal de la pierna, y en las acciones compensatorias proximales. No se encontraron diferencias significativas en la velocidad y longitud del paso, entre pacientes que llevaban estas férulas y aquellos que utilizaban zapatos ordinarios. Los autores indicaron la influencia significativa de las AFO en la flexión de cadera (en la fase oscilante) y en la dorsiflexión del tobillo. Esto permite disminuir la caída del pie y reducir la frecuencia de tropiezos (22).

El estudio de Uygur (23) se centró en la población pediátrica. Los 55 participantes utilizaron una AFO nocturna para mantener y reforzar la posición neutral y el tobillo en ángulo de $90^{\circ}$. A pesar de no proporcionar datos de resultado o de seguimiento, los autores concluyen que se debe evitar que los infantes utilicen una férula, antes de que su uso sea necesario. 
Refiriéndonos a un grupo de CMT concreto, en las personas con tipo $1 \mathrm{~A}$, el uso de ortesis AFO elásticas mejora la economía articular durante la marcha, reduciendo el coste energético por unidad de distancia. Esto se demuestra en un menor nivel de esfuerzo metabólico y una mejoría en la eficiencia del movimiento, en comparación con el uso único de zapatos ortopédicos (24).

\section{Calzado Ortopédico}

Más allá de las cuestiones de elección personal, relacionadas con el estilo o color de los zapatos, las personas con CMT deben considerar su calzado como un dispositivo más del tratamiento, que puede mejorar la estabilidad y el equilibro, en bipedestación y durante la marcha. La mejor elección es un zapato firme, pero a la vez, flexible, que permita realizar las modificaciones requeridas para ajustarse al pie (2).

Al principio, puede ser suficiente el uso de calzado ordinario, de una talla mayor, que permita insertar plantillas de gel para amortiguar el paso y dar soporte a la vez. Se debe revisar el interior de los zapatos y eliminar cualquier mínimo elemento externo (como pequeñas piedras) que puedan lesionar los tejidos cutáneos y que pasen desapercibidos a la persona, debido a la disminución de su sensibilidad (2).

Cuando la evolución del nivel de afectación aumenta, el uso de zapatos ortopédicos, en combinación con los estiramientos aplicados en fisioterapia, es una buena primera opción para el tratamiento del dolor asociado, la caída del pie y la prevención de esguinces. Esto permite disminuir las alteraciones de la marcha y aumentar la distancia de desplazamiento (25).

\section{Recomendaciones para el uso de calzado en CMT}

A continuación, se exponen algunos consejos en relación con el uso de calzado ortopédico, que derivan del consenso y la experiencia profesional (26):
- Se debe evitar el uso de zapatos abiertos, por el riesgo de lesión de dedos o talones. Los pies siempre irán protegidos.

- Los zapatos han de permitir un espacio suficiente para los dedos de los pies y, a su vez, el apoyo del talón y el arco. Se recomienda que exista media pulgada entre el dedo del pie más largo y el borde del zapato.

- En relación a su material, la parte superior ha de ser suave y flexible, mientras que su revestimiento debe ser liso, libre de costuras y arrugas.

- No se aconseja utilizar los mismos zapatos varios días seguidos. Cuando son nuevos, es importante ir aumentando su uso progresivamente, para evitar dolor y daños en la piel, por lo que se debe vigilar diariamente su aspecto y la presencia de lesiones.

- Mantener una hidratación adecuada de los pies, pero también realizar un completo secado cuando se encuentran húmedos, para prevenir la infección.

- Para las zonas callosas, se recomienda el uso de aceite de coco o jojoba, para evitar las grietas, pero no aplicarlo entre los dedos de los pies.

- Los calcetines han de cambiarse una vez al día, recomendándose que sean de fibras naturales.

- El corte de las uñas de los pies también es relevante, ya que puede ser causa de lesiones importantes o infecciones. Por ello, esta tarea ha de dejarse en manos de un podólogo.

- Este profesional también será el encargado de valorar las callosidades y la presencia de signos de exceso de presión. 
- Las variaciones extremas de temperatura son nocivas, por lo que se tratará de que ésta sea constante, y evitar el uso de bolsas de agua caliente y la exposición de las piernas a la luz solar prolongada.

- Por último, se hace especial hincapié en el mantenimiento y vigilancia de la circulación vascular, especialmente del retorno venoso. Por ello, se evitará cualquier elemento que comprima los tejidos cutáneos o restrinja el movimiento, los agentes vasoconstrictores como el tabaco, y las posturas estáticas e inadecuadas, como cruzar las piernas.

\section{Conclusiones}

Las enfermedades de CMT provocan limitaciones muy variables en las personas con este diagnóstico, pero en general, se caracterizan por pérdida de fuerza muscular, inestabilidad y presencia de deformidades en MMII.

Estas complicaciones se abordan mediante estrategias rehabilitadoras aplicadas por un equipo interdisciplinar, con el uso de ortesis y/o calzado ortopédico y, en algunos casos, cirugía.

Las ortesis más utilizadas en dicha enfermedad son: tobilleras, AFO y KAFO, siempre adaptadas a las características del usuario.

Para el uso de calzado ortopédico es necesario tener en cuenta una serie de consideraciones y/o recomendaciones que garanticen una buena adaptación y adecuación a las condiciones de la persona.

La finalidad última de la utilización de estos procedimientos es garantizar el mayor grado de autonomía personal posible y mejorar la calidad de vida del usuario. 


\section{References} 1. AFM-Téléthon, ASEM F. Enfermedades de personas que sufren de pie caído. Universidad Católica Charcot-Marie-Tooth. Federación ASEM, editor. Barcelona; Popular de Risaralda; 2008.

2002.

17. Wegener C, Wegener K, Smith R, Schott K-H, Burns 2. Muscular Dystrophy Association. Charcot-Marie- J. Biomechanical effects of sensorimotor orthoses in adults Tooth Disease (CMT) [Internet]. 2017. Available from: with Charcot-Marie-Tooth disease. 2015;6-8.

https://www.mda.org/disease/charcot-marie-tooth $\quad 18$ Guillebastre B, Calmels P, Rougier PR. Assessment 3. Laurá M, Neurologist C, Reilly MM. Charcot of Appropriate Ankle-Foot Orthoses Models for Patients with Marie-Tooth disease (CMT). J Peripher Nerv Syst. Charcot-Marie-Tooth Disease. Am J Phys Med Rehabil. 2011 2011;Mar 16(1):1-14. Aug;90(8):619-27.

4. Charcot-Marie-Tooth Association. ¿Qué es la 19. Corrado B, Ciardi G, Bargigli C. Rehabilitation enfermedad de Charcot-Marie-Tooth (CMT)? [Internet]. Management of the Charcot-Marie-Tooth Syndrome: A 2015. Available from: Systematic Review of the Literature. Medicine (Baltimore). https://www.cmtausa.org/understanding-cmt/what-is-cmt/ 2016 Apr;95(17):e3278.

5. AFM-Telethon, Federación ASEM. Avances en la 20. Rose KJ, Raymond J, Refshauge K, North KN, Burns enfermedad de Charcot-Marie-Tooth. Barcelona: J. Serial night casting increases ankle dorsiflexion range in Federación ASEM; 2014. children and young adults with Charcot-Marie-Tooth disease:

6. AFM-Telethon, ASEM F. Enfermedades a randomised trial. J Physiother. 2010;56(2):113-9.

Neuromusculares -49 fichas-. Federación ASEM, editor. 21. Refshauge KM, Raymond J, Nicholson G, van den Barcelona; 2003. Dolder PA. Night splinting does not increase ankle range of 7. Ornstein H, Stilwell G. The importance of Physical motion in people with Charcot-Marie-Tooth disease: A Activity [Internet]. Charcot-Marie-Tooth Association. 2015. randomised, cross-over trial. Aust J Physiother. Available from: https://www.cmtausa.org/resource- 2006;52(3):193-9.

center/treatment-management/exercise/ 22. Ramdharry GM, Day BL, Reilly MM, Marsden JF. 8. Federación ASEM, AFM-Telethon. Uso de ortesis Foot drop splints improve proximal as well as distal leg y enfermedades neuromusculares. Barcelona: Federación control during gait in Charcot-Marie-Tooth disease. Muscle ASEM; 2011. Nerve. 2012;512-9.

9. Pousada García T, Sánchez-Ortiz Muñoz M, 23. Uygur F, Bek N, Kürklu B, Yilmaz Ö. Orthotic Gómez-Serranillos L, Pérez Laguía M. Un servicio para la management of the lower limb in children with hereditary promoción de la autonomía de las personas con motor sensory neuropathy (HMSN). Prosthet Orthot Int. enfermedades neuromusculares en la Federación ASEM. 2001;25:139-43.

Rev Española Discapac. 2013;I(I):215-23. 24. Menotti F, Laudani L, Damiani A, Mignogna T, 10. Misener DB, Rayner F. Overview \& Orthotic Macaluso A. An anterior ankle-foot orthosis improves Management of Charcot Marie Tooth. In Glenolden, walking economy in Charcot-Marie-Tooth type 1A patients. Pensilvania: Charcot-Marie-Tooth Association; 2015. Prosthet Orthot Int. 2014 Oct;38(5):387-92.

11. Charcot-Marie-Tooth Association. Charcot-Marie- 25. Bensoussan L, Jouvion A, Kerzoncuf M, Delarque Tooth and Your Feet [Internet]. [cited 2017 May 12]. A, Theodoridou E, Milhe de Bovis V, et al. Orthopaedic shoes Available from: https://www.cmtausa.org/resource- along with physical therapy was effective in Charcot-Mariecenter/treatment-management/foot-care/

Tooth patient over 10 years. Prosthet Orthot Int. 2016 12. Eagle Michelle. Guidelines for exercise and Oct;40(5):636-42.

orthoses in children with neuromuscular disorders. London: 26. Charcot-Marie-Tooth Association. Important Foot Muscular Dystrophy Campain; 2003.

Care for People with CMT [Internet]. Available from: 13. Vinci P, Gargiulo P. Poor compliance with ankle- https://www.cmtausa.org/resource-center/treatmentfoot-orthoses in Charcot-Marie-Tooth disease. Eur J Phys management/foot-care/important-foot-care-for-people-withRehabil Med. 2008;1(44):27-31.

14. Ramdharry GM, Pollard AJ, Marsden JF, Reilly MM. Comparing Gait Performance of People with CharcotMarie-Tooth Disease Who Do and Do Not Wear Ankle Foot Orthoses. Physiother Res Int [Internet]. 2012 Dec 1 [cited 2017 Sep 22];17(4):191-9. Available from: http://doi.wiley.com/10.1002/pri.531

15. Noriega F. Guía del paciente: Charcot Marie Tooth. IICOP; 2015. 